\title{
Two-Stage Kalman Filter for Fault Tolerant Estimation of Wind Speed and UAV Flight Parameters
}

\author{
Chingiz Hajiyev ${ }^{1}$, Demet Cilden-Guler ${ }^{2}$, Ulviye Hacizade ${ }^{3}$ \\ ${ }^{I}$ Istanbul Technical University, Faculty of Aeronautics and Astronautics, Istanbul, Turkey, cingiz@itu.edu.tr \\ ${ }^{2}$ Istanbul Technical University, Faculty of Aeronautics and Astronautics, Istanbul, Turkey \\ ${ }^{3}$ Halic University, Faculty of Engineering, Istanbul, Turkey
}

\begin{abstract}
In this study, an estimation algorithm based on a two-stage Kalman filter (TSKF) was developed for wind speed and Unmanned Aerial Vehicle (UAV) motion parameters. In the first stage, the wind speed estimation algorithm is used with the help of the Global Positioning System (GPS) and dynamic pressure measurements. Extended Kalman Filter (EKF) is applied to the system. The state vector is composed of the wind speed components and the pitot scale factor. In the second stage, in order to estimate the state parameters of the UAV, GPS, and Inertial Measurement Unit (IMU) measurements are considered in a Linear Kalman filter. The second stage filter uses the first stage EKF estimates of the wind speed values. Between these two stages, a sensor fault detection algorithm is placed. The sensor fault detection algorithm is based on the first stage EKF innovation process. After detecting the fault on the sensor measurements, the state parameters of the UAV are estimated via robust Kalman filter (RKF) against sensor faults. The robust Kalman filter algorithm, which brings the fault tolerance feature to the filter, secures accurate estimation results in case of a faulty measurement without affecting the remaining good estimation characteristics. In simulations, noise increment and bias type of sensor faults are considered.
\end{abstract}

Keywords: Unmanned aerial vehicle, Kalman filter, fault detection, wind speed, GPS, pitot tube.

\section{INTRODUCTION}

A popular approach to the estimation of the flight parameters of a small Unmanned Aerial Vehicle (UAV) includes the integration of sensor measurements from a Global Positioning System (GPS) with that of a low-cost Inertial Navigation System (INS). Various formulations of flight parameter estimation using GPS/INS sensor fusion exist in the literature [1]-[4], containing comparison studies evaluating different estimation algorithms.

In this work, the wind field is estimated for both horizontal and vertical wind using GPS and pitot tube measurements. Estimation of the wind field is useful in UAV applications for various objectives such as dropping objects, target tracking, automatic control, trajectory optimization, and air traffic control [5]. There is some existing work in the area of wind estimation. A Kalman-like filter is derived in [6] for wind velocity estimation based on magnetic heading, true airspeed, and radar measurements. This filter is called the velocity bias filter for wind estimation. In [7], the problem of aircraft wind velocity estimation is performed using the aircraft dynamic response rather than the wind triangle relationship. In this method, it is assumed that the mathematical model of the aircraft is perfectly known. The primary limitation of these types of wind estimation methods is the requirement of a known aircraft model. This can be very limiting for some types of aircraft where the model has not yet been derived, or additional system uncertainties have been introduced.

In [8], a pitot-tube-independent estimation for airspeed is presented using the Extended Kalman Filter (EKF). This paper focuses on developing an approach to fault detection for airspeed sensors in UAVs by using data from gyros, accelerometers, GPS, and wind vanes. Based on the kinematics model of the UAV, an estimator is proposed to provide analytical redundancy using information from the above-mentioned sensors. The $\chi^{2}$ test and cumulative sum detector are employed to detect the occurrence of airspeed measurement faults together.

In [9], an estimator is proposed based on the kinematics model of the UAV in order to provide analytical redundancy using information from gyros, accelerometers, GPS, and wind vanes. This filter process is independent of the airspeed measurement and the aircraft dynamics model. The estimation of the wind velocity is necessary to relate ground velocity to airspeed, which is why the wind velocity components are considered as states.

Aircraft are usually equipped with vanes or multi multi-port air data probes that, when properly calibrated, can be used to infer the wind velocity, angle-of-attack (AOA), and side-slip 
angle (SSA). In this study the wind velocity, AOA and SSA are estimated using standard sensors. The standard sensor suite contains GPS, Inertial Measurement Unit (IMU), and a pitot-static tube.

In this study, an estimation algorithm based on a two-stage Kalman filter (TSKF) was developed for wind speed and UAV motion parameters. In the first stage, the wind speed estimation EKF was developed by the usage of GPS measurements and dynamic pressure measurements. In the second stage, the estimation of the state parameters of the UAV was made based on the GPS and IMU measurements by using the Linear Kalman filter. The second stage filter uses the first stage EKF estimates of the wind speed values. Between these two stages, a sensor fault detection algorithm is designed. The innovation sequence of the wind speed estimation filter is used for fault detection purposes.

A robust Kalman filter (RKF) algorithm, which makes the filter fault-tolerant and keeps giving accurate estimation results in case of faulty measurements, is proposed in this paper. This filter is designed in order to minimize the effects of sensor faults and to estimate the UAV flight parameters accurately. After detecting the fault on the sensor measurements, the state parameters of the UAV are estimated by using RKF against sensor faults.

\section{STATEMENT OF THE PROBLEM}

This work aims to estimate aircraft body-axis velocity components $(u, v, w)$, Euler attitude angles $(\phi, \theta, \psi)$, and three-axis wind velocity components $\left(\mu_{N}, \mu_{E}, \mu_{D}\right)$. This estimation is performed through the functional fusion of IMU measurements of attitude angles $(\phi, \theta, \psi)$, angular rates $(p, q, r)$, GPS measurements of velocity components $\left(V_{x}, V_{y}, V_{z}\right)$, and the dynamic pressure measurements by the pitot-static tube $\left(P_{d}\right)$. Due to the random nature of wind, e.g. turbulence, it is challenging to predict the behavior of the local wind field dynamics. Therefore, the wind velocity state dynamics are modeled as a random walk process [9],

$$
\left[\begin{array}{l}
\dot{\mu}_{N}(t) \\
\dot{\mu}_{E}(t) \\
\dot{\mu}_{D}(t)
\end{array}\right]=w_{p}(t)
$$

where $w_{p}$ denotes the zero-mean Gaussian process noise vector with the covariance matrix $Q_{p}$. The UAV state equation is given in a discrete-time linear state-space format,

$$
X(k+1)=F X(k)+B U(k)+G W(k)
$$

where $X(k)$ is the vector of the system state, $F$ is the transition matrix of the system, $B$ is the control distribution matrix, $U$ is the control input vector, which comprises the control surface deflections and wind velocity, $W$ is the system noise vector with a covariance matrix $Q, G$ is the transition matrix of system noise.
The body-axis velocity components can be rotated into the Earth-fixed frame using the transformation matrix $A(\phi, \theta, \psi)$

$$
A(\phi, \theta, \psi)=\left[\begin{array}{ccc}
\cos \psi & -\sin \psi & 0 \\
\sin \psi & \cos \psi & 0 \\
0 & 0 & 1
\end{array}\right]\left[\begin{array}{ccc}
\cos \theta & 0 & \sin \theta \\
0 & 1 & 0 \\
-\sin \theta & 0 & \cos \theta
\end{array}\right]\left[\begin{array}{ccc}
1 & 0 & 0 \\
0 & \cos \phi & -\sin \phi \\
0 & \sin \phi & \cos \phi
\end{array}\right]
$$

and corrected for wind by [5],

$$
\left[\begin{array}{l}
V_{N}^{G P S} \\
V_{E}^{G P S} \\
V_{D}^{G P S}
\end{array}\right]=A(\phi, \theta, \psi)\left[\begin{array}{l}
u \\
v \\
w
\end{array}\right]+\left[\begin{array}{l}
\mu_{N} \\
\mu_{E} \\
\mu_{D}
\end{array}\right]+v^{G P S}
$$

As an Earth-fixed frame, the North-East-Down (NED) frame is used. This is a reference frame locally fixed to a point on the Earth's surface and its X-Y-Z axes pointing to NorthEast-Down. The Z-axis aligns the Earth's ellipsoid normal direction. In (3) $V_{N}^{G P S}, V_{E}^{G P S}, V_{D}^{G P S}$ are the NED ground velocity measurement components by GPS, $v^{G P S}$ is the zeromean Gaussian measurement noise vector of GPS.

It is required to estimate the wind speed, Air Data System (ADS) scale factor and UAV states in the presence of sensor faults on the acquired information above.

\section{TWO-STAGE ESTIMATION OF THE WIND SPEED AND UAV STATES}

A two-stage Kalman filter is proposed for wind speed and UAV state estimation purposes. In the first stage, the wind speed is estimated by the EKF using GPS and pitot tube measurements. Here, the wind speed components and pitot scale factor are considered as state vector variables. In the second stage, the estimation of the state parameters of the UAV was made based on the GPS and IMU measurements by using the Linear Kalman filter. The second stage filter uses the first stage EKF estimates of the wind speed values. Between these two stages, a sensor fault detection algorithm is designed. After detecting the fault in the sensor measurements, the state parameters of the UAV are estimated via the robust Kalman filter against sensor faults. Fault tolerance and accurate estimations are achieved by using the RKF algorithm in case of faulty measurements. The interaction of two filtering stages is shown in the structural diagram of Fig.1.

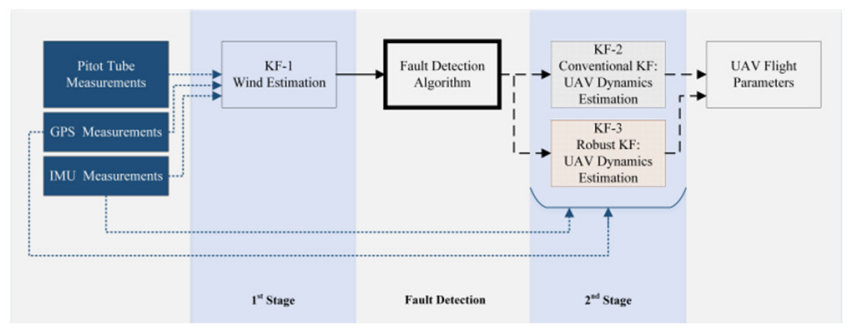

Fig.1. General block diagram of the proposed two-stage estimation procedure. 


\section{A. Design of the Kalman filter for wind velocity estimation}

Nonlinear state-space formulations of wind velocity estimation problems of the unmanned aerial vehicle are discussed in this section. As the formulations use the relationship of the wind triangle, it is necessary to have the knowledge of both the ground and airspeed. The formulations use the pitot-static tube airspeed and the GPS velocity estimates. Here, the state vector includes north $\left(\mu_{N}\right)$, east $\left(\mu_{E}\right)$, down $\left(\mu_{D}\right)$ components of the wind velocity and the ADS scale factor $(\zeta)$. The effects of the sideslip angle, angle-of-attack and the air density parameters can be estimated by using the scale factor. The state-space system is composed of the state vector $x[10]$,

$$
x=\left[\begin{array}{llll}
\mu_{N} & \mu_{E} & \mu_{D} & \zeta
\end{array}\right]^{T}
$$

The dynamic pressure measurements by the pitot-static tube $P_{d}$ are presented as output. As there is no information on the state dynamics, dynamic equations are expressed by random walk process,

$$
x(k)=x(k-1)+w_{p}(k-1)
$$

where $w_{p}$ is the zero-mean Gaussian system noise vector with the process covariance matrix $Q_{p}$. Output vector can be composed by using the wind triangle relationship

$$
\vec{V}_{\text {air }}=\vec{V}_{\text {ground }}-\vec{V}_{\text {wind }}
$$

where $\vec{V}_{\text {air }}, \vec{V}_{\text {ground }}, \vec{V}_{\text {wind }}$ are the air, ground, and wind velocity vectors in the NED coordinate system, respectively. After taking the square of each side of the equation (6) it can be obtained [10]

$$
V_{\text {air }}^{2}=\left(V_{N}-\mu_{N}\right)^{2}+\left(V_{E}-\mu_{E}\right)^{2}+\left(V_{D}-\mu_{D}\right)^{2}
$$

The airspeed of the pitot-static tube $\left(V_{\text {pitot }}\right)$ can be expressed in terms of total airspeed $\left(V_{\text {air }}\right)$, angle-of-attack $(\alpha)$, and sideslip angle $(\beta)$,

$$
V_{\text {pitot }}=V_{\text {air }} \cos \alpha \cos \beta
$$

Dynamic pressure can be written considering the Bernoulli equation as:

$$
P_{d}=\frac{\rho}{2} V_{p i t o t}^{2}
$$

where $\rho$ represents the air density. So, the scale factor $(\zeta)$ can be written as:

$$
\zeta=\frac{\rho}{2}(\cos \alpha)^{2}(\cos \beta)^{2}
$$

If all of these are combined, the output vector equation becomes:

$$
P_{d}=\zeta\left[\left(V_{N}-\mu_{N}\right)^{2}+\left(V_{E}-\mu_{E}\right)^{2}+\left(V_{D}-\mu_{D}\right)^{2}\right]+v_{p}
$$

where $v_{p}$ is the zero-mean Gaussian measurement noise with the variance $R_{p}$.

The ground speed components are measured by GPS and therefore the equations for the ground speed are

$$
\left[\begin{array}{c}
V_{N}^{G P S} \\
V_{E}^{G P S} \\
V_{D}^{G P S}
\end{array}\right]=\left[\begin{array}{c}
V_{N} \\
V_{E} \\
V_{D}
\end{array}\right]+v^{G P S}
$$
[11],

The linear state model can be written in the following form

$$
x(k+1)=A x(k)+G w_{p}(k)
$$

where $\mathrm{A}$ and $\mathrm{G}$ are $4 \times 4$ unit matrices.

The measurement equation (11) is nonlinear and can be written in the form:

$$
z(k)=h[x(k), k]+v_{p}(k)
$$

where $h[\cdot]$ is the nonlinear measurement function, $x(k)$ is 4-dimensional state vector at time $k$. It is assumed that both noise vectors $w_{p}(k)$ and $v_{p}(k)$ are Gaussian white noise with zeroing,

$$
E\left[w_{p}(k)\right]=E\left[v_{p}(k)\right]=0, \quad \forall k
$$

Filter algorithm based on the described system and measurements in (13)-(14) can be given. The estimation of states (4) can be found based on the EKF. The estimation value is,

$$
\hat{x}(k / k)=\hat{x}(k / k-1)+K(k)\{z(k)-h[\hat{x}(k / k-1, k)]\}
$$

The extrapolation value from the dynamic function can be found as,

$$
\hat{x}(k / k-1)=A \hat{x}(k-1 / k-1)
$$

Filter-gain of the EKF is,

$$
\begin{aligned}
& K(k)=P(k / k-1) \nabla h^{T}[\hat{x}(k / k-1), k] \times \\
& \left\{\nabla h[\hat{x}(k / k-1), k] P(k / k-1) \nabla h^{T}[\hat{x}(k / k-1), k]+R_{p}(k)\right\}^{-1}
\end{aligned}
$$

where $\nabla h[\hat{x}(k / k-1), k]=\frac{\partial h[\hat{x}(k / k-1), k]}{\partial \hat{x}(k / k-1)}$ is the partial derivative of the measurement function with respect to the states.

The covariance matrix of the extrapolation error is, 
$P(k / k-1)=A P(k-1 / k-1) A^{T}+G Q_{p}(k-1) G^{T}$

The covariance matrix of the filtering error is,

$$
P(k / k)=[I-K(k) \nabla h[\hat{x}(k / k-1), k]] P(k / k-1)
$$

where $I$ is the identity matrix.

The innovation sequence is presented as,

$$
\Delta(k)=z(k)-h[\hat{x}(k / k-1), k]
$$

The innovation covariance is,

$$
P_{\Delta}(k)=\nabla h[\hat{x}(k / k-1), k] P(k / k-1) \nabla h^{T}[\hat{x}(k / k-1), k]+R_{p}(k)
$$

EKF that estimates the state vector of the system is expressed with the formulas (16) - (22).

\section{B. Design of the Kalman filter for UAV state estimation}

Let us consider the UAV flight dynamics described by the linear state equation (2) using the trim condition and measurement equation

$$
Z(k)=H(k) X(k)+V(k)
$$

where $Z(k)$ is the measurement vector, $H(k)$ is the measurement matrix, $V(k)$ is the random vector of measurement noise. Assume that the random vectors $W(k)$ and $V(k)$ are Gaussian white noise with zero mean. Note that $\{W(k)\}$ and $\{V(k)\}$ are assumed mutually uncorrelated.

The UAV state vector is

$$
X=\left[\begin{array}{lllllllll}
u & v & w & \phi & \theta & \psi & p & q & r
\end{array}\right]^{T}
$$

The measurement vector is expressed as

$Z=\left[\begin{array}{lllllllll}V_{N}^{G P S} & V_{E}^{G P S} & V_{D}^{G P S} & \phi_{m} & \theta_{m} & \psi_{m} & p_{m} & q_{m} & r_{m}\end{array}\right]^{T}$

As seen from equation (3), the ground velocity measurement components $V_{N}^{G P S}, V_{E}^{G P S}, V_{D}^{G P S}$ are nonlinear with respect to the UAV states. Through converting, we can also change the measurements to be

$$
Z=\left[\begin{array}{lllllllll}
u & v & w & \phi_{m} & \theta_{m} & \psi_{m} & p_{m} & q_{m} & r_{m}
\end{array}\right]^{T}
$$

where

$$
\left[\begin{array}{l}
u \\
v \\
w
\end{array}\right]=A^{-1}\left(\phi_{m}, \theta_{m}, \psi_{m}\right)\left[\begin{array}{l}
V_{N}^{G P S}-\hat{\mu}_{N} \\
V_{E}^{G P S}-\hat{\mu}_{E} \\
V_{D}^{G P S}-\hat{\mu}_{D}
\end{array}\right]
$$

In this case, the measurement noise variances of the indirect measurements $(u, v, w)$ should be calculated. As a result, the measurement nonlinearities are eliminated and for estimation of the UAV state vector (24), the linear KF can be used. The input vector is

$$
U=\left[\begin{array}{lllllll}
\delta_{c} & \delta_{t} & \delta_{w} & \delta_{p} & \hat{\mu}_{N} & \hat{\mu}_{E} & \hat{\mu}_{D}
\end{array}\right]^{T}
$$

The first four elements of the input vector (28) represent the control surface deflections (column, throttle, wheel, and pedal, respectively) and the last three elements of the wind velocity estimations. For estimation of the state vector of UAV (24), the linear Kalman filter can be used [11].

Using the second stage estimation results $(\hat{u}, \hat{v}, \hat{w})$, the airspeed $\left(V_{\text {air }}\right)$, angle-of-attack $(\alpha)$, and sideslip angle $(\beta)$ can be calculated

$$
\hat{V}_{a i r}=\sqrt{\hat{u}^{2}+\hat{v}^{2}+\hat{w}^{2}}
$$

$$
\hat{\alpha}=\arctan \left(\frac{\hat{w}}{\hat{u}}\right)
$$

$$
\hat{\beta}=\arcsin \left(\frac{\hat{v}}{\sqrt{\hat{u}^{2}+\hat{w}^{2}+\hat{v}^{2}}}\right)
$$

\section{Sensor fault detection}

A sensor fault detection algorithm is considered based on the normalized innovation squared [12]

$$
\beta(k)=\tilde{\Delta}(k)^{2}
$$

where $\tilde{\Delta}(k)$ is the normalized innovation sequence of the Kalman filter. This statistical function (32) has $\chi^{2}$ distribution with $s=1$ degree of freedom, where $s$ is the dimension of the innovation vector. Now consider the following two hypotheses:

$$
\begin{aligned}
& H_{0}: \text { Sensor fault occurs } \\
& H_{1}: \text { No sensor faults. }
\end{aligned}
$$

If the level of significance, $\alpha$, is selected as,

$$
P\left\{\chi^{2}>\chi_{\alpha, s}^{2}\right\}=\alpha ; 0<\alpha<1
$$

the threshold value $\chi_{\alpha, s}^{2}$ can be found. Hence, when the hypothesis $H_{1}$ is true, the value of the statistical function $\beta(k)$ will be greater than the threshold value $\chi_{\alpha, s}^{2}$ i.e.:

$$
\begin{aligned}
& H_{0}: \beta(k) \leq \chi_{\alpha, s}^{2} \quad \forall k \\
& H_{1}: \beta(k)>\chi_{\alpha, s}^{2} \quad \exists k .
\end{aligned}
$$




\section{ROBUST KALMAN FILTER FOR UAV STATE ESTIMATION}

In normal operation conditions, the Kalman filter gives sufficiently good estimation results where any kind of measurement malfunction is not observed. However, when the measurements are faulty, filter estimation outputs become inaccurate. Therefore, a robust Kalman filter algorithm, which has the ability to be fault-tolerant with accurate estimation results in case of a faulty measurement with no effect on the remaining good estimation characteristics, is introduced.

The base of the RKF is the comparison of real and theoretical values of the covariance of the innovation sequence [12]. When the operational condition of the measurement system mismatches with the models used in the synthesis of the filter, then the Kalman filter gain changes according to the differentiation in the covariance matrix of the innovation sequence. Under these circumstances, the covariance matrix of the innovation sequence differs as:

$$
P_{\Delta}(k)=H(k) P(k / k-1) H^{T}(k)+S(k) R(k)
$$

and the Kalman gain becomes

$$
\begin{aligned}
& K(k)=P(k / k-1) H^{T}(k) \times \\
& {\left[H(k) P(k / k-1) H^{T}(k)+S(k) R(k)\right]^{-1}}
\end{aligned}
$$

where $P(k / k-1)$ is the covariance matrix of the extrapolation error, $R(k)$ is the covariance matrix of the measurement noise, $S(k)$ is the measurement noise scale factor. $S(k)$ can be written as

$$
S(k)=\frac{\Delta^{T}(k) \Delta(k)-\operatorname{tr}\left\{H(k) P(k / k-1) H^{T}(k)\right\}}{\operatorname{tr}\{R(k)\}}
$$

Here $\operatorname{tr}\{\cdot\}$ denotes the trace of the related matrix. In the case of malfunction in the measurement system, the adaptation of the Kalman filter is performed by correcting the Kalman gain automatically. If there is a sensor fault in the system, it brings an increase in the scale factor $S(k)$. Higher $S(k)$ causes a smaller Kalman gain because of the covariance of the innovation sequence which is also increased in the robust case. Consequently, small Kalman gain value reduces the effect of the faulty innovation sequence on the state estimation process. In all other cases, where sensors operate normally, scale factor takes the value of $S(k)=1$ and the filter runs conventionally.

\section{TSKF SIMULATION RESULTS}

The wind speed and longitudinal and lateral motion parameters of UAV are estimated using the proposed TSKF. In order to test the algorithm, the GPS receiver and pitot tube faults are examined. In simulations two types of faults are taken into consideration: noise increment and continuous bias.

\section{A. GPS fault case}

First, noise increment type of fault was adopted and the measurement equation was redefined for the interval between 300 and 500 seconds as

$$
V^{G P S}(k)=V(k)+5 v^{G P S}(k)
$$

Simulation results are given in Fig.2. to Fig.4. Estimation results of the wind speed are given in Fig.2. In this figure, the blue line shows the actual values and the red line - estimated values. The obtained results show that the wind speed estimations converge to their actual values except for the faulty period and UAV states can be estimated with high accuracy.

The fault detection algorithm works using the statistic (32). The level of significance in the sensor fault detection is chosen as 0.05 . The threshold value, $\chi_{\alpha, s}^{2}$ for $\alpha=0.05$ and $s=1$ is found as $\chi_{\alpha, s}^{2}=2.71$. By using the algorithm, the faulty period is detected between 300 and 500 seconds as seen in Fig.3. by using $\beta(k)$, fault detection statistics.

Second, continuous bias type of GPS measurement fault is considered and the measurement equation is redefined for the interval between 300 and 500 seconds as

$$
V^{G P S}(k)=V(k)+v^{G P S}(k)+4
$$

Estimation errors of wind speed are presented in Fig.4. As seen from the figure, the estimation errors of the proposed TSKF are small except for the faulty period.
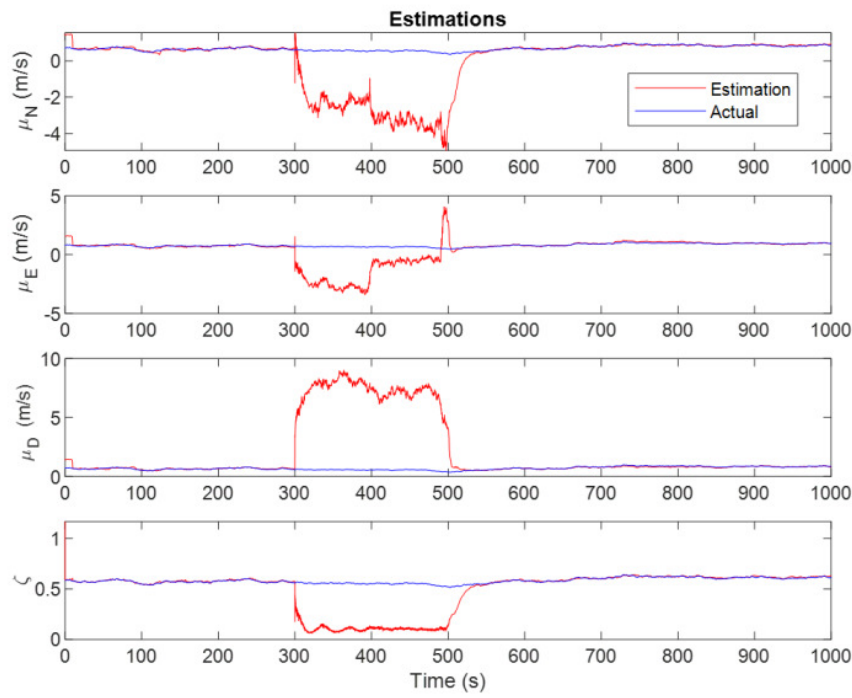

Fig.2. Wind speed and scale factor estimation results: actual value - blue line; estimated value - red line (GPS noise increment case). 


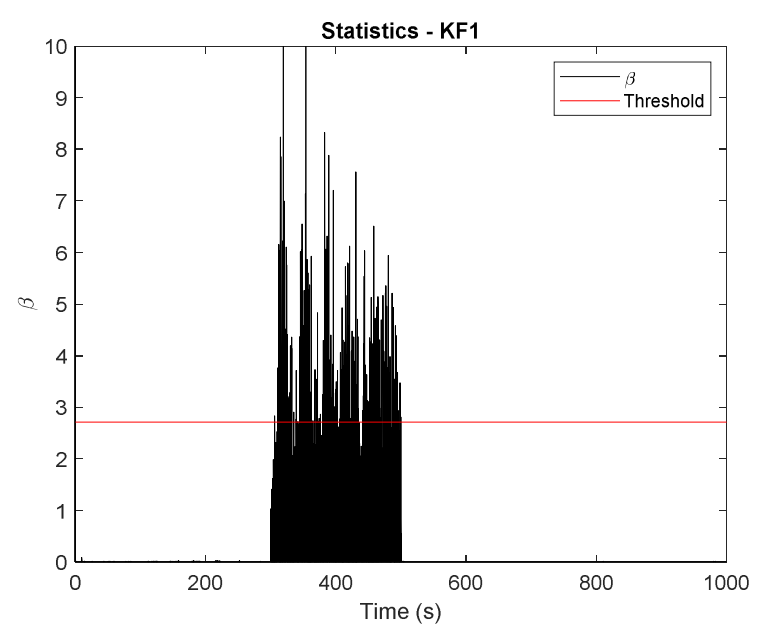

Fig.3. Fault detecting statistics (GPS noise increment case).
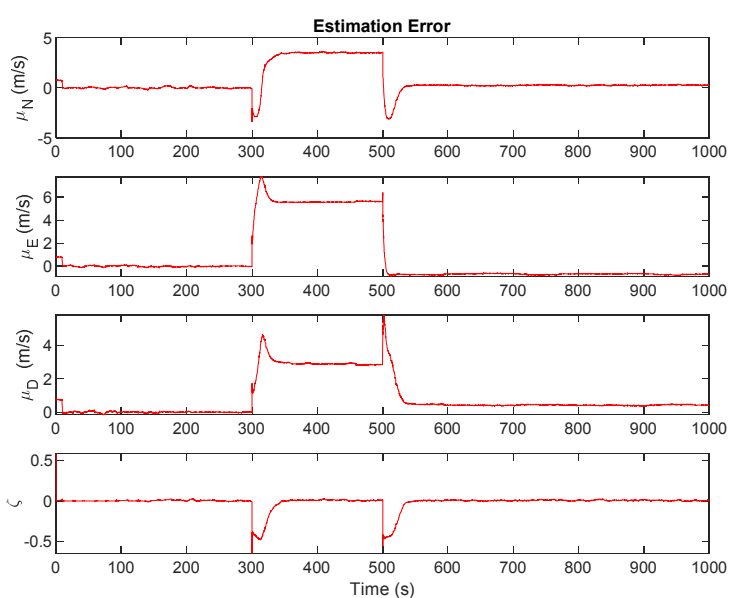

Fig.4. Wind speed and scale factor estimation errors (GPS bias case).

\section{B. Pitot-tube fault case}

The same two fault cases are applied to the pitot-tube measurements. Noise increment type of fault was adopted and the measurement equation was redefined for the interval between 300 and 500 seconds as,

$$
z(k)=h[x(k), k]+5 \times v_{p}(k)
$$

In Fig.5., wind speed components and scale factor estimation errors are shown, while Fig.6. represents the fault detection statistics. As can be seen, the faulty pitot-tube measurements are detected by the algorithm just like GPS. The faulty period can be seen clearly in both figures, which means that the estimations of the conventional filter were deteriorated, but the fault detection algorithm comes into play and detects the whole period of the fault.

Second, continuous bias type of measurement fault is considered and the measurement equation is redefined for the interval between 300 and 500 seconds as,

$$
z(k)=h[x(k), k]+v_{p}(k)+3
$$

Similar results are found for the pitot-tube faults as shown in Fig.7. with wind estimations.
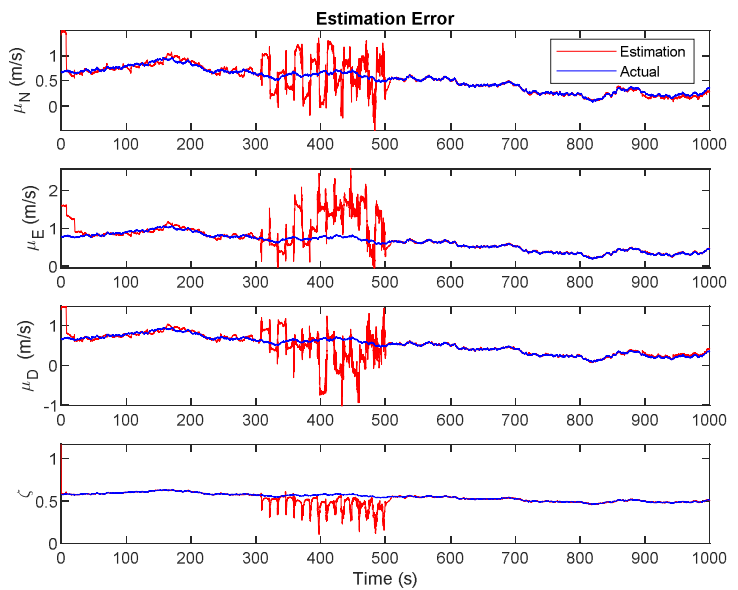

Fig.5. Wind speed and scale factor estimation results: actual value - blue line; estimated value - red line (Pitot-tube noise increment case).

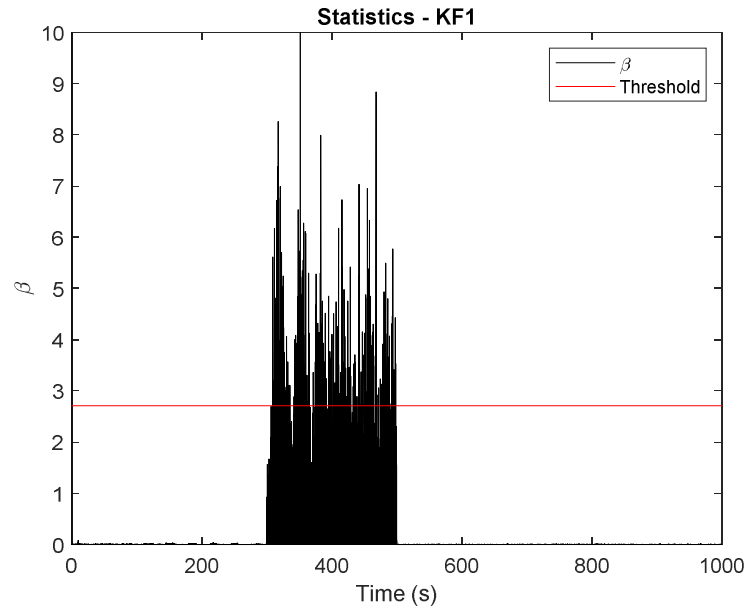

Fig.6. Fault detecting statistics (Pitot-tube noise increment case).
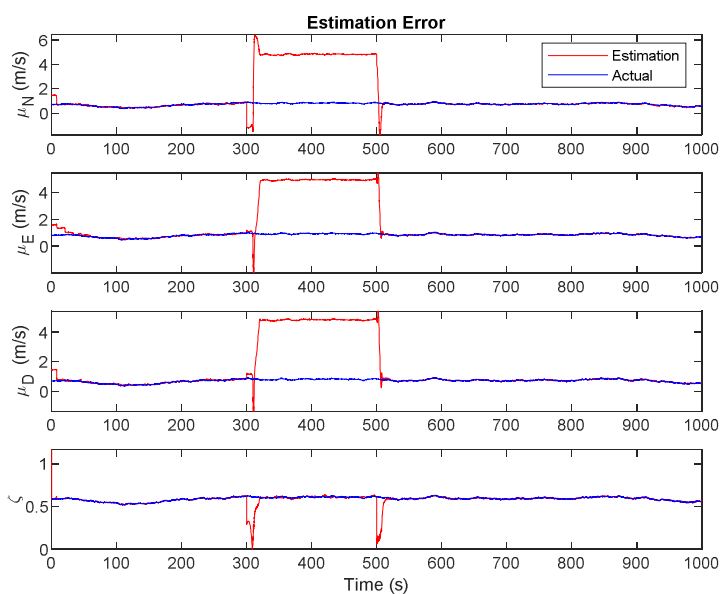

Fig.7. Wind speed and scale factor estimation results: actual value - blue line; estimated value - red line (Pitot-tube bias case). 


\section{Robust TSKF simulation results}

The wind speed and longitudinal and lateral motion parameters of UAV are also estimated via presented robust TSKF in case of the defined faulty conditions. In order to test the algorithm, all types of faults are applied to the GPS and the pitot-tube measurements between 300 and 500 seconds. Estimation errors of wind speed and UAV's velocity components in the presence of noise increment fault on GPS measurements are presented in Fig.8. and Fig.9., respectively. As seen from the figures, the estimation errors of the proposed robust TSKF are significantly better than the results of non-robust TSKF. Similar results were obtained for each scenario (Table 1.). In Table 1., mean errors of the TSKF and robust TSKF estimations are presented in terms of wind velocity components for each case. As can be seen from the table, the adaptive structure of the robust filter improves the accuracy of the results in every scenario.
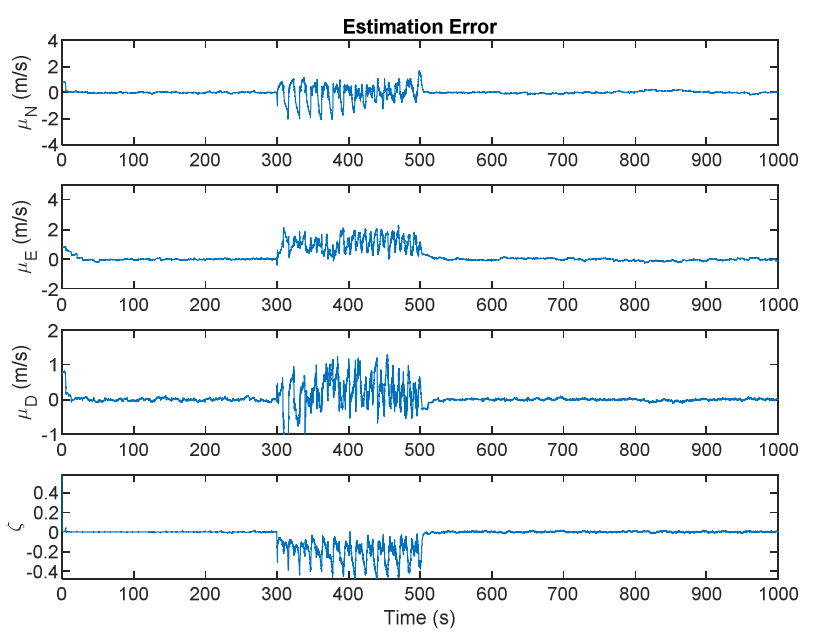

Fig.8. Wind speed estimation errors (robust - noise increment).
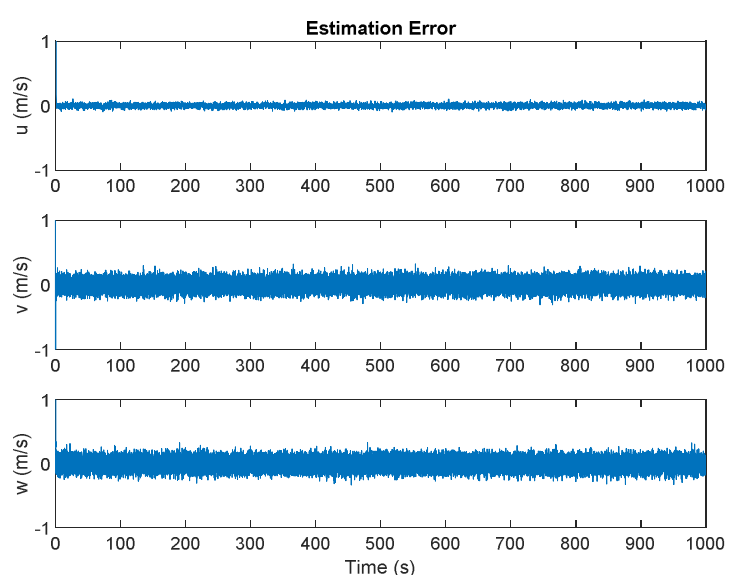

Fig.9. Estimation errors of the velocity components (robust - noise increment).
Table 1. Wind estimation mean errors for the sensor faults.

\begin{tabular}{|l|l|c|c|c|c|c|c|}
\hline \multirow{2}{*}{$\begin{array}{l}\text { Sensor } \\
\text { Type }\end{array}$} & Type of & \multicolumn{3}{|l|}{ TSKF } & \multicolumn{3}{|c|}{ Robust TSKF } \\
\cline { 2 - 8 } & Fault & $\mu_{N}$ & $\mu_{E}$ & $\mu_{D}$ & $\mu_{N}$ & $\mu_{E}$ & $\mu_{D}$ \\
\hline \multirow{2}{*}{ GPS } & $\begin{array}{l}\text { Noise } \\
\text { Increment }\end{array}$ & 3.06 & 2.91 & 5.63 & 0.06 & 1.68 & 0.82 \\
\cline { 2 - 8 } & Bias & 3.26 & 4.12 & 2.12 & 2.67 & 3.86 & 1.65 \\
\hline \multirow{2}{*}{$\begin{array}{l}\text { Pitot- } \\
\text { Tube }\end{array}$} & $\begin{array}{l}\text { Noise } \\
\text { Increment }\end{array}$ & 0.28 & 0.18 & 0.40 & 0.04 & 0.07 & 0.12 \\
\cline { 2 - 8 } & Bias & 0.70 & 1.42 & 1.83 & 0.06 & 0.54 & 0.24 \\
\hline
\end{tabular}

\section{CONCLUSIONS}

In this work a two-stage Kalman filter was developed for wind speed and UAV state estimation. In the first stage, the wind speed estimation algorithm is developed by the usage of GPS and dynamic pressure measurements. For this purpose, the Extended Kalman Filter based on nonlinear measurements was designed. The wind speed components and pitot scale factor are estimated by EKF using GPS and pitot tube measurements. In the second stage, the estimation of the state parameters of the UAV dynamic model was done by using the Conventional Linear KF based on the GPS and IMU measurements and the first stage EKF estimates of the wind speed values.

The fault detection algorithm using the first stage filter's innovation sequence is put between these two stages in order to give information about the faults to the second filter and if any fault occurs then the filter should switch to a robust TSKF. By implementing this sub-step, fault tolerance is achieved by mitigating the fault effects and improving the estimation results in case of a fault. The proposed method also allows calculating air velocity, angle-of-attack, and the sideslip angle. These parameters are estimated using only standard sensors (GPS, IMU, and pitot-static tube).

In simulations, two types of sensor faults are considered: noise increment and continuous bias. Simulation results show that the proposed robust TSKF mitigates the effects of sensor faults and estimates the wind speed and UAV flight parameters accurately.

\section{ACKNOWLEDGEMENTS}

This research is part of the project numbered with TM3061 which is fully financed by Turkish Aerospace Inc. In order to learn more details about the project, please apply/refer to Turkish Aerospace Inc.

\section{REFERENCES}

[1] Hu, G., Gao, S., Zhong, Y. (2015). A derivative UKF for tightly coupled INS/GPS integrated navigation. ISA Transactions, 56, 135-144.

[2] Nguyen, H.-D., Nguyen, V.-H., Nguyen, H.-V. (2017). Tightly-coupled INS/GPS integration with magnetic aid. In 2nd International Conference on Control and Robotics Engineering (ICCRE). IEEE, 207-212.

[3] Nourmohammadi, H., Keighobadi, J. (2018). Fuzzy adaptive integration scheme for low-cost SINS/GPS navigation system. Mechanical Systems and Signal Processing, 99, 434-449. 
[4] Hajiyev, C., Kamiloglu, D. (2018). Design of looselycoupled INS/GPS integration system applied to Boeing747 aircraft model. International Journal of Sustainable Aviation, 4 (3/4), 163-177.

[5] Rhudy, M., Larrabee, T., Chao, H., Gu, Y., Napolitano, M. (2013). UAV attitude, heading, and wind estimation using GPS/INS and an air data system. In AIAA Guidance Navigation and Control Conference. AIAA 2013-5201.

[6] Lefas, C.C. (1987). Real-time wind estimation and tracking with transponder downlinked airspeed and heading data. IEEE Transactions on Aerospace and Electronic Systems, AES-23 (2), 169-174.

[7] Lee, J.H., Sevil, H.E., Dogan, A., Hullender, D. (2013). Estimation of maneuvering aircraft states and timevarying wind with turbulence. Aerospace Science and Technology 31 (1), 87-98.

[8] Guo, D., Zhong, M., Zhou, D. (2018). Multisensor datafusion-based approach to airspeed measurement fault detection for unmanned aerial vehicles. IEEE Transactions on Instrumentation and Measurement, 67 (2), 317-327.

[9] Guo, D., Zhong, M., Zhou, D. (2018). Multisensor datafusion-based approach to airspeed measurement fault detection for unmanned aerial vehicles. IEEE Transactions on Instrumentation and Measurement, 67 (2), 317-327.
[10] Cho, A., Kim, J., Lee, S., Kee, C. (2011). Wind estimation and airspeed calibration using a UAV with a single-antenna GPS receiver and pitot tube. IEEE Transactions on Aerospace and Electronic Systems, 47 (1), 109-117.

[11] Rhudy, M.B., Gu, Y., Gross, J.N., Chao, H. (2017). Onboard wind velocity estimation comparison for unmanned aircraft systems. IEEE Transactions on Aerospace and Electronic Systems, 53 (1), 55-66.

[12] Hajiyev, C., Cilden-Guler, D., Hacizade, U. (2019). Two-stage Kalman filter for estimation of wind speed and UAV flight parameters based on GPS/INS and pitot tube measurements. In 9th International Conference on Recent Advances in Space Technologies (RAST). IEEE, $875-880$

[13] Hajiyev, C. (2007). Adaptive Filtration Algorithm with the filter gain correction applied to integrated INS/Radar altimeter. Proceedings of the Institution of Mechanical Engineers, Part G: Journal of Aerospace Engineering, 221 (5), 847-885.

Received October 16, 2019 Accepted January 20, 2020 